\title{
Commentary: upper airways dysfunction
}

\author{
J R Stradling
}

These three case reports from Liverpool, Grenoble, and Dublin provide some insights into the respiratory function of the upper airway, both when awake and asleep. The respiratory control of the larynx and pharynx is extremely complicated. This is probably for several reasons, not least because the upper airway has other functions to perform apart from being an adequate air conduit and protector of the lower air passages. The pharyngeal and laryngeal muscles receive both tonic (postural) and phasic (respiratory) inputs. The muscular output is a combination of these inputs to the anterior horn cells, and hence the fall in postural muscle tone with sleep onset (and more so during REM sleep) can render these muscles less able to perform their respiratory duties, particularly if other factors are conspiring to threaten the upper airway.

Interestingly, not all the respiratory activity of the upper airway is designed to maintain an open, low resistance, air path. Hypoxia can lead to a reduction in laryngeal dilator activity leaving adductor activity (usually used to oppose the cords for speech) unopposed, a response thought to delay expiration thus keeping lung volumes raised and providing nature's own PEEP. Loss of this laryngeal braking mechanism in neonates - for example, following intubation - can produce considerable hypoxaemia.

Although excessive fat in the neck is the usual cause of obstructive sleep apnoea (OSA), there are many others. This means that the finding of obstructive apnoeas during sleep should lead to a search for the actual cause or diagnosis - just as anaemia is not a diagnosis, but a finding requiring explanation. The absence of a large neck should lead to a search for other causes, the more commonly encountered being retrognathia, hypothyroidism, large tonsils, acromegaly, and mucopolysacharridoses.

The case reports by Doherty et al and Pépin et al remind us that there are yet more causes of OSA to consider. The Arnold-Chiari malformation essentially interferes with brainstem function by mechanical compression and interference with blood supply. As such it can interfere with the protective reflexes of the upper airway, both the afferent and efferent pathways. Both obstructive and central sleep apnoea have been reported. In the case report by Doherty et al it appears that upper airways obstruction was the only component since, with a tracheostomy, the patient's nocturnal breathing was normal. His level of obstruction was pharyngeal, although laryngeal sleep apnoea has been reported in this condition. The authors suggest that loss of pharyngeal sensation was an important component of the obstructive sleep apnoea since we know there are important pharyngeal dilator mechanisms activated by subatmospheric pressures in the pharynx, and possibly by the vibration of snoring itself. Given the relatively extensive interference with brainstem function it is difficult to be sure whether loss of protective reflexes or loss of dilator muscle activity was the real cause. Extensive surface anaesthesia in subjects who snore worsens the degree of obstruction but does not produce gross OSA as was present in this case.

The case report by Pépin et al describes OSA in association with rheumatoid arthritis. There are a number of ways in which rheumatoid disease could cause OSA, and again this case report cannot be definitive above the actual cause. The patient's rheumatoid disease had caused subluxation of C3-4 with pyramidal signs, and destruction of the temporomandibular joint had resulted in retropositioning of the mandible. It is unlikely that brainstem damage occurred, with reduced pharyngeal dilator activity, without other brainstem signs. Other causes considered were the sleep disruption due to joint pain since experimental sleep fragmentation reduces dilator activity of the upper airway to a small extent.

A possible cause not mentioned is laryngeal sleep apnoea due to destruction of the arytenoid bones in the larynx and thus the dilator action of the posterior cricoarytenoid muscles. In the one case we have seen this presented with stridor at night rather than snoring (as occurs in OSA associated with the Shy-Drager syndrome and laryngeal weakness). The history is not always useful in differentiating the two noises but can be.

The third case report by Shiels et al describes a laryngeal problem which often disappears with sleep, perhaps giving a clue to its functional nature. This report describes a 17 year old girl with cystic fibrosis and probable asthma who presented with inspiratory stridor and hoarseness of voice. Bronchoscopic examination showed a falling together of the vocal cords during inspiration rather than the usual dilation. The conventional expiratory pulmonary function tests were surprisingly normal, but inspiratory flow-volume loops showed upper airways obstruction. The authors suggest that the abnormality was functional in nature. Such dysfunction is more common in young women with some health care background. It is also more common in patients with asthma, which again may be because of more knowledge of health matters, or could it, in fact, be related to bronchospasm? There is some evidence that laryngeal narrowing can occur as part of obstructive airways disease, perhaps through activation of certain reflexes. Thus, laryngeal stridor may not always be due to psychological problems, which of course introduces uncertainty into the management of these patients
Osler Chest Unit The Church Headington, Oxford OX3 7LJ, UK

J R Stradling

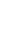

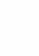


at a time when a firm diagnosis would be most helpful. There is a suggestion that measurement of blood gases and lung volumes may help, with the finding of hyperventilation but no hyperinflation suggesting a functional cause. However, it is recommended that, despite suspicions that an individual's acute wheeze or stridor is factious, they should initially be given full asthma treatment. When evidence has built up, and reassuring patterns of rapid recovery are established, then one can reduce the emergency treatment next time and show that it is not necessary to achieve equally rapid recovery. Once confident of the diagnosis, a referral to a skilled speech therapist can be very helpful, along with psychiatric referral if appropriate.

It is also possible to get variable laryngeal function following partial damage to recurrent laryngeal nerves. We recently saw a patient who presented initially with variable inspiratory stridor due to bilateral cord adduction. The real diagnosis (a subaortic arch lung tumour) only became clear when there was progression to left sided permanent cord paralysis which prompted a computed tomographic scan of the thorax. It appears that early in the process of recurrent laryngeal nerve compression or infiltration there can be differential effects on the fibres, and hence on adductor and abductor function. The bilateral effects are difficult to explain initially but may be due to stimulation of sensory fibres in the nerve with reflex laryngeal narrowing.

These three case reports indicate how important it is for chest physicians to know about the larynx; unfortunately it is sometimes regarded as a bit "too far north" to be of interest and more in the territory of the ENT surgeon.

\section{Dieulafoy's disease of the bronchus}

\author{
M Sweerts, A G Nicholson, \\ P Goldstraw, B Corrin
}

\begin{abstract}
Dieulafoy's vascular malformation has not been described outside the gastrointestinal tract. Two cases are reported in which this vascular abnormality arose in right lower lobe bronchi, both of which presented with massive haemoptysis.

(Thorax 1995;50:697-698)
\end{abstract}

Keywords: Dieulafoy's disease, bronchus.

Dieulafoy's disease is an abnormality of the gastric or, less frequently, the intestinal arteries, characterised by wide mucosal branches that are susceptible to ulceration and bleeding. ${ }^{1-3}$ This abnormality has not been reported in the respiratory tract, but we have recently encountered two patients who each had such a vascular anomaly in a bronchus and presented in middle age with massive haemoptysis.

\section{Case reports}

CASE 1

A 35 year old woman, a non-smoker, was admitted with massive haemoptysis. In the previous 14 years she had had numerous similar but less severe episodes, usually preceded by an upper respiratory tract infection. Repeated examination of the nose and throat and multiple bronchoscopies had not revealed the source of the bleeding. Pulmonary arteriography, chest radiography, computed tomographic scans, and a ventilation-perfusion scan had all been normal. However, bronchoscopy had shown clot confined to the right lower lobe bronchus on four occasions, suggesting that a local abnormality was responsible for the haemorrhage. It had also been noted that she had unusually long index fingers and first metacarpal bones.

On this admission bronchoscopic examination revealed a pulsating bleeding point in the right lower lobe bronchus. Diathermy and selective bronchial embolisation failed to control the haemorrhage and a right lower lobectomy was performed. Postoperative recovery was uneventful and four months later the patient has had no further haemoptysis.

CASE 2

A 59 year old retired male publican, who smoked 40 cigarettes and drank 10 units of alcohol a day, was admitted to another hospital as an emergency with haemoptysis (or haematemesis) and melaena. There was no history of any previous such haemorrhage. Endoscopic examination showed no gastrointestinal abnormalities and he was transferred to the Royal Brompton Hospital for control of the haemoptysis. There was no evidence of a coagulopathy and bronchoscopic examination revealed that the right lower lobe bronchus was filled with blood. A right lower and middle lobectomy was performed. No follow up data are available.

\section{HISTOLOGY}

The histological picture was similar in the two cases. In both, a large artery showing focal thinning of the media extended between the 